\title{
MITOCHONDRIAL DISEASE THERAPY
}

\author{
KANIKA KHAJURIA ${ }^{1}$, VIJAY KHAJURIA ${ }^{2 *}$, VINEETA SAWHNEY²
}

${ }^{1}$ Department of Pharmacology, ASCOMS, Sidhra, Jammu, (UT) Jammu and Kashmir, India. ${ }^{2}$ Department of Pharmacology, Government Medical College, Kathua, (UT) Jammu and Kashmir, India. Email: vijaykhaj@yahoo.co.in

Received: 05 February 2021, Revised and Accepted: 30 March 2021

ABSTRACT

Mitochondria perform number of important functions, including synthesis of adenosine triphosphate (ATP) and generation of reactive oxygen species (ROS). Most of the organs depend on ATP to perform. Therefore, in depleted or dysfunctional mitochondrial states, there is less energy production coupled with the accumulation of oxidants. Oxidative stress is involved in the pathophysiology of various disorders especially involving neurons and the cardiovascular system. Mitochondrial diseases are a clinically heterogeneous group of disorders resulting from either inherited or spontaneous mutations in mitochondrial deoxyribonucleic acid (mtDNA) or nuclear DNA. In primary mitochondrial dysfunction disease, the mutation affects the oxidative phosphorylation (OXPHOS) functioning, while secondary mitochondrial dysfunction does not involve OXPHOS genes. Since mutations of genes are involved, therefore, therefore the mitochondrial dysfunctional states are not easy to treat. However, number of strategies that lead to increase ATP production, counter ROS facilitates improvement. The current strategy is to focus on stimulating the biogenesis of mitochondria, antioxidants, and cofactors to enhance ATP synthesis. The role of non-pharmaceuticals cannot be underestimated either. The exercise, diet, and environment influence have well-established beneficial outcome in these disorders. Gene therapy holds promise in the future management of these complex disorders.

Keywords: Mitochondrial disorders, Treatment, Energy metabolism, Coenzyme Q10, Thiamine, Riboflavin, Carnitine, Arginine, Bezafibrate, Resveratrol, Gene therapy.

(C) 2021 The Authors. Published by Innovare Academic Sciences Pvt Ltd. This is an open access article under the CC BY license (http://creativecommons.org/ licenses/by/4.0/) DOI: http://dx.doi.org/10.22159/ajpcr.2021v14i5.41016. Journal homepage: https://innovareacademics.in/journals/index.php/ajpcr

\section{INTRODUCTION}

Mitochondrial diseases are a clinically heterogeneous group of disorders due to mutation affecting either mitochondrial deoxyribonucleic acid (DNA) or nuclear DNA [1]. These disorders are of two types. Primary mitochondrial disease has mitochondrial or nuclear DNA mutation affecting oxidative phosphorylation (OXPHOS) functioning, while secondary mitochondrial dysfunction (SMD) does not involve OXPHOS genes [2]. These disorders are not easy to treat [3]. However, diet, exercise, and specific vitamin supplements have a role in their management [4]. It is also of utmost importance to avoid mitochondrial toxic drugs such as valproic acid (VPA) and aminoglycosides.

\section{PHARMACOLOGICAL INTERVENTION}

Foremost among therapeutic interventions include CO-FACTORS OF OXPHOS for promoting adenosine triphosphate (ATP) production.

Coenzyme Q10 (CoQ10 as ubiquinone) is an essential electron carrier in OXPHOS process for ATP production. It is known to diminish with advancing age and result in the production of less ATP. Its deficiency is found to be autosomal recessive in inheritance and associated with five major clinical phenotypes encephalopathy, severe infantile multisystemic disease, cerebellar ataxia, isolated myopathy, and nephrotic syndrome. Primary CoQ10 deficiency occurs when there is a mutation of genes involved in its biosynthesis. Respiratory chain defects, reactive oxygen species (ROS) production, and apoptosis contribute toward primary CoQ10 deficiency. While in secondary deficiency, biosynthesis genes are not affected [5].

CoQ10 is not water-soluble and has low bioavailability and this causes a delay in attaining peak concentrations. It has shown to be beneficial in mitochondrial dysfunctional states. CoQ10 and its analogs restore electron flow and increase mitochondrial antioxidant capacity [6].

Its supplementation increases energy production and reduces fatigue [7]. It also has strong antioxidant properties [8]. Dietary
CoQ10 causes improvement in exercise capacity and as such is useful in cardiovascular conditions such as hypertension, heart failure, and improves endothelial functions [9]. It is useful in neurodegenerative conditions such as Alzheimer's disease (AD), Parkinsonism, and Huntington's disease [10]. CoQ10 supplementation also benefits muscle pains in patients associated with statins therapy [11].

CoQ10 is one of the most widely used natural supplement. Dose in the pediatric group is $2-8 \mathrm{mg} / \mathrm{kg} /$ day while in adults, it ranges between 50 and $600 \mathrm{mg} /$ day. CoQ10 is a safe option in the treatment of mitochondrial states. Meat, poultry, fish, soybeans, canola oils, and nuts are the rich sources of CoQ10. Fruits, vegetables, eggs, and dairy products are moderate sources of CoQ10.

MitoQ or mitoquinone is an orally active, water-soluble antioxidant that helps in mitochondrial dysfunction. It reduces mitochondrial overproduction of ROS and has high bioavailability and attains peak levels quickly in body. It does not need to be taken with food unlike CoQ10 [12].

In Mito $\mathrm{Q}$ the lipophilic triphenylphosphonium cation is bound to ubiquinone moiety of co-enzyme Q10 [13,14]. Once targeted to the matrix, ubiquinone is reduced to ubiquinol that facilitates electron transfer between complexes I/II to complex III and acts as an antioxidant by decreasing lipid peroxidation, MitoQ10 has been found to improve endothelial nitric acid (NO) bioavailability and blood pressure [9]. Addition of MitoQ10 to treatment with Angiotensin receptor blocker (losartan) is beneficial against target organ damage development in hypertension [15].

It improves electron transport chain (ETC) function and protects cardiolipin which is a mitochondrial phospholipid found exclusively at the inner mitochondrial membrane. It has therapeutic potential for $A \beta$ - and oxidative stress-associated neurodegenerative disorders, particularly Alzheimer's disease (AD) [16].

MitoTEMPOL is a combination of the antioxidant piperidine nitroxide. TEMPOL with the lipophilic cation triphenyl phosphonium gives 
MitoTEMPOL the ability to pass through lipid bilayers with ease and accumulate several hundred-fold in mitochondria. Its antioxidant action detoxifies superoxide by cycling between its nitroxide and oxoammonium forms, as well as oxidizing ferrous iron to limit hydroxyl radical formation [17].

Mito TEMPOL effectively reduces ROS production in both mitochondrial and cytosolic compartments in failing myocytes in animal studies [18]. Interestingly, this compound has been shown to be protective against oxidative injury in various pathologies, such as endotoxin-induced liver injury, sepsis-induced acute kidney injury, hypertension, or colitis [19-22].

\section{NUTRACEUTICALS}

Nutraceuticals are derived from food products. They are not traditionally recognized nutrients but have positive physiological effects on the body and have potential in the management of dyslipidemic states [23]. These are either vitamins or metabolic supplements. Nutraceuticals commonly used are thiamine (Vitamin B1), riboflavin (Vitamin B2), nicotinamide (Vitamin B3), dichloroacetate, creatine monohydrate, carnitine, L-arginine, or vitamin combinations.

All nutraceuticals increase the biological activity of complexes in the ETC. Enhancement of nitric oxide (NO) production is useful in mitochondrial encephalomyopathies, lactic acidosis, and stroke-like episodes (MELAS), cystamine (in Huntington's disease).

\section{Riboflavin}

It is a water-soluble B Vitamin $\left(\mathrm{B}_{2}\right)$ and is a precursor of flavoprotein. Riboflavin plays an important part in the number of metabolic pathways. Its active derivatives, the flavin mononucleotide and flavin adenine dinucleotide, ensure the functions of numerous flavoprotein, including dehydrogenases, oxidases, monooxygenases, and reductases. They play a pivotal role in mitochondrial ETC, $\beta$-oxidation of fatty acids, redox homeostasis, citric acid cycle, branched-chain amino acid catabolism, chromatin remodeling, DNA repair, protein folding, and apoptosis.

Number of inborn errors of flavin metabolism and flavoenzyme functions such as multiple acyl-CoA dehydrogenation deficiency (MADD) and riboflavin transporter deficiencies respond to riboflavin supplementation $[24,25]$.

Alteration in riboflavin levels will result in mitochondrial dysfunction and reduced energy levels will cause disorders such as neurodegenerative, cataract, and cardiovascular diseases. Mutations of mitochondrial DNA or nuclear DNA encoding for flavin transporters and flavoenzyme in mitochondrial dysfunction are associated with neurological disorders [26]. Riboflavin treatment has been also tested in children with complex II (or succinate dehydrogenase) deficiency [27].

Arginine (2-amino-5-guanidinovaleric acid) is an amino acid used in the biosynthesis of proteins. It is an immediate precursor of nitric oxide (NO) and is also necessary for the synthesis of important biological molecules such as creatine, ornithine, polyamines, agmatine, proline, glutamate, dimethyl-arginine, and urea. NO is an important neurotransmitter and possesses vasodilator properties.

Mitochondria in their dysfunctional states are unable to generate sufficient ATP for body needs. In MELAS syndrome, which is a primary mitochondrial disorder, there is ample evidence of deficiency of NO, $\mathrm{L}$ arginine, and cyclic guanosine monophosphate due to endothelial dysfunction resulting from impaired blood perfusion. It contributes to stroke-like episodes, myopathy, and lactic acidosis in MELAS. Since arginine and citrulline both are NO precursors; they increase NO production and therefore, their administration alleviates endothelial dysfunction and helps in managing mitochondrial disorders [28].

Administration of L-arginine $(500 \mathrm{mg} / \mathrm{kg} /$ dose $)$ decreases the severity of stroke-like symptoms, enhances the dynamics of microcirculation, and reduces tissue injury from ischemia in patients with MELAS [29].

\section{Folinic acid}

Folinic acid a reduced form of folic acid is a water-soluble B Vitamin $\left(B_{9}\right)$. It is a cofactor in multiple metabolic reactions. ATP-driven folate transporter causes folate to enter brain through choroid plexus cells. Kearns-Sayre syndrome (KSS) which is a mitochondrial DNA syndrome, there is a reduce folate transport into brain due depletion of ATP, resulting in low spinal fluid (CSF) 5-methyltetrahydrofolate (5MTHF) levels. Early treatment with high-dose folinic acid is effective in the treatment of KSS [30].

Patients with mitochondrial complex I encephalomyopathies also present with low 5MTHF level and respond to treatment with folinic acid in addition to supplementation with radical scavengers and cofactors of deficient respiratory enzymes [31]. Folinic acid supplementation is of use in some patients of mitochondrial disease [32].

\section{Nicotinamide adenine dinucleotide (NAD)}

NADH is a substrate for enzymes and cofactor in more than 200 cellular redox reactions. NADH has a vital role in ETC as it delivers electron from metabolite hydrolysis to transport chain in mitochondria. NADH possesses antioxidant properties and such is used to neurodegenerative disorders such as Parkinson's disease (PD) and Alzheimer's disease (AD), where basic pathology is oxidative damage [33,34]. In chronic fatigue, $\mathrm{NADH}$ is also reported to reduce symptoms [35]. The most preferred supplementation of NADH is orally in its precursor forms such as niacin, nicotinic acid, or nicotinamide. Conventionally, prime indication of NADH remains pellagra, where it is deficient and is a disease of four D's (dermatitis, diarrhea, dementia, and ultimately death).

Another therapeutic target is the stimulation of NAD biosynthetic pathway which increases protein deacetylation through SIRT and improves mitochondrial biogenesis [36]. Similarly, in animal trials, it delayed the progression to diabetic cardiomyopathy [37]. Nicotinamide riboside has been reported to effectively delay early and late-stage myopathy progression by robustly inducing mitochondrial biogenesis in skeletal muscle and brown adipose tissue, preventing mitochondrial abnormalities and mtDNA deletion formation [38].

Membrane phospholipids - dietary phospholipids (PL) can be employed to replace the damaged oxidized membrane mitochondrial lipids to enhance its function [39]. Phospholipids and antioxidants combination has been found useful in chronic fatigue, aging, and fibromyalgia syndrome [40].

Carnitine transports fatty acids into mitochondria for beta-oxidation and increases ATP production. It is, therefore, very useful in reducing exercise-induced fatigue as fat is utilized during extreme physical effort. Carnitine also modulates coenzyme A and removes excessive acyl groups from body [41]. Carnitine deficiency or impaired fatty acid oxidation is associated with reduced mitochondrial function, insulin resistance, and coronary heart disease, sepsis, and renal disease [41]. In aging, where there is a reduced mitochondrial OXPHOS leading to increased oxidants and reduced energy production, supplementation of carnitine corrects these adverse events. L-carnitine is found to have cardiac beneficial potential in patients of heart failure $[42,43]$ through decreased ROS production. Dose in the pediatric group is orally $0.1 \mathrm{~g} / \mathrm{kg} / \mathrm{d}$, while in adults, it is $5 \mathrm{~g} / \mathrm{d}$ orally.

Polyphenols (flavolons, theaflavin, and epicatechin) have antioxidant actions and found to be useful in long-standing disorders, including cardiovascular diseases [44]. Polyphenols are naturally occurring compounds rich in red wine, green tea, olive oil, and dark chocolate. Mitochondrial pathways are involved in cardioprotective action of some flavonoids [45].

Quercetin is a plant pigment (flavonoid) found in green tea, apples, berries, Ginkgo biloba, St. John's Wort, American elder, and buckwheat tea has a large amount of quercetin. It increases endothelial NO synthase activity and hemeoxygenase-1 [46]. Quercetin also has significant antioxidant properties [47]. 
Polyphenols of olive oil and red wine reduce intracellular ROS levels [48], while epicatechin of green tea lowers the expression of proinflammatory molecules [49].

\section{DRUGS WITH ANTIOXIDANT ACTIVITY}

Vitamins $\mathrm{C}$ and $\mathrm{E}$ are the most popular antioxidant therapy in chronic diseases. However, their role in mitochondrial dysfunction in not clearly substantiated as mitochondria absorb only a small amount of these vitamins or they may interact with other therapeutic agents [50]. ROS scavengers and Vitamin K3, lipoic acid, triacetyluridine, melatonin, $\mathrm{N}$-acetylcysteine are effective antioxidants and are quite effective in mitochondrial disorders, carvedilol though a beta-blocker has been found useful in heart failure because of its antioxidant properties [51].

ACE inhibitors and angiotensin receptor-II blockers improve mitochondrial dysfunction. Captopril in animal studies is found to increase mitochondrial biogenesis, while losartan restored mitochondrial dysfunction and kidney damage through preservation of glutathione and SOD activity [52]. Statins (atorvastatin and simvastatin) have antioxidative stress potential and reduced the activity of mitochondrial NO synthase, cytochrome c release, and lipid peroxidation [53]. Oral antidiabetic thiazolidinediones (rosiglitazone) reduce lipid oxidation and hinders the development of atherosclerosis through up-regulation of peroxisome proliferator-activated receptor (PPAR $\gamma$ ) [54]. Even the mainstay drug of diabetes the metformin, is found to reduce mitochondrial ROS production, enhances the activity of antioxidant enzymes and decreases inflammation attributed to I/R injury beneficial in diabetic cardiomyopathy.

Inhibition of the mitochondrial permeability and transition pore (mtPTP) and apoptosis. Cyclosporine, nortriptyline, and dimebon are the inhibitors of mtPTP and apoptosis. These are beneficial in mitochondrial dysfunctional conditions such as $\mathrm{AD}, \mathrm{PD}$, acute kidney injury, and myocardial infarction $[55,56]$.

\section{ENHANCEMENT OF MITOCHONDRIAL BIOGENESIS}

\section{Sirtuin analogs}

SIRT1 protects against inflammation and oxidative damage and is involved in many cellular processes such as regulation of glucose and lipid metabolism, insulin signaling in the liver, adipose tissue, and skeletal muscles.

Sirtuins (SIRT) are a family of NAD-dependent deacylases comprised of SIRT I to7. Sirtuins affect the activity of multiple metabolic enzymes through stimulation of the PPAR family, PPAR signaling regulates gene expression of multiple metabolic pathways. These lead to beneficial effects of exercise, lean diets and found to even delay aging. In advancing age, obesity, sedentary habits, the NAD, and sirtuin level levels decline, while exercise has beneficial effects. SIRT3, SIRT4, and SIRT5 localize to mitochondria and regulate targets involved in biochemical pathways. Mitochondrial sirtuin has an important role in aging and age-related diseases $[57,58]$.

In eukaryotes, mitochondria carry out numerous functions central to cellular and organismal health. How mitochondrial activities are regulated in response to different environmental conditions, such as variations in diet, remains an important unsolved question in biology. Here we review emerging evidence suggesting that reversible acetylation of mitochondrial proteins on lysine residues represents a key mechanism by which mitochondrial functions are adjusted to meet environmental demands. In mammals, three members of the sirtuin class of NAD+-dependent deacetylases - SIRT3, SIRT4, and SIRT5 - localize to mitochondria and regulate targets involved in a diverse array of biochemical pathways. The importance of this activity is highlighted by recent studies of SIRT3, indicating that this protein suppresses the emergence of diverse age-related pathologies: hearing loss, cardiac fibrosis, and malignancy. Together, these findings argue that mitochondrial protein acetylation represents a central means by which mammals regulate mitochondrial functions to maintain cellular and organismal homeostasis.
Resveratrol is found in grapes and red wine, is an antioxidant, an apoptosis inhibitor, and an SIRT-1 agonist. Resveratrol has a potential role in the treatment of diabetes, cardiovascular disease, neurodegenerative disease, cancer, obesity, and aging. Its effects as antioxidant are associated with an induction of genes for OXPHOS and mitochondrial biogenesis and decrease in PGC- $1 \alpha$ acetylation and an increase in PGC- $1 \alpha$ activity.

Cardiomyocyte protective action of resveratrol is because it inhibits ROS generation in cardiomyocytes. Resveratrol also increases mitochondrial contents in endothelial cells [29-63]. Resveratrol restores mitochondrial functions and decreases insulin resistance. Hypoglycemia observed with resveratrol is because of a decrease in ROS and an increase in glucagon-like peptide [64].

Resveratrol also exhibits glioprotective action in azide-produced oxidative damage [65].

Bezafibrate is a hypolipidemic drug and a PPAR agonist increase mitochondrial transcription, mtRNA synthesis, and biogenesis [66]. Its short-term use has been shown to reduce number of complex IVimmunodeficient muscle fibers by inducing mitochondrial biogenesis and improved cardiac function. However, there are concerns about its long-term complications [67].

Taurine is a non-essential amino acid and is important for protein translation in mitochondria and has a therapeutic role in mitochondrial disorders $[68,69]$. Its therapy provides a source of substrate for the taurine conjugation reaction and restores mitochondrial protein biosynthesis, thereby improving mitochondrial function and reducing ROS generation [70].

MELAS has a defective taurine modification of the mutant mt tRNAs, which result in a deficiency in protein synthesis. Taurine orally reduces the recurrence of stroke-like episodes and increases taurine modification in mitochondrial RNA in MELAS [71]. It also possesses neuroprotective in nickel toxicity by reducing oxidative stress and restores mitochondrial function [72].

\section{REDUCING MITOCHONDRIAL FISSION AND ENHANCING MITOCHONDRIAL FUSION}

Normally mitochondria constantly undergo fission and fusion and there is a balance between two. In neurodegenerative diseases such as AD, PD, and Huntington's disease, the fragmentation takes the upper hand due to increased oxidation and generation of ROS, resulting in mitochondrial structural changes and dysfunction and cell damage. Hence, there is always an effort to develop inhibitors of mitochondrial fission.

Normal mitochondrial fission is regulated and maintained by two GTPase genes: Fis1 and Dynamin-related protein 1 (Drp1), effectively influences cell survival and apoptosis by mediating the mitochondrial fission process, which is an intricate process regulating both cellular and organ dynamics, including development, apoptosis, acute organ injury, and various diseases. It is suggested that Drp1 can reduce mitochondrial fission and elevate mitochondrial fusion [73].

Mammalian target of rapamycin complex 1 controls protein synthesis. It is a cytosolic Ser/Thr kinase belonging to the phosphatidylinositol kinase-related protein kinases family is a regulator of many cellular processes, including mRNA translation, and is a vital regulator in mitochondrial functions and increase ATP production capacity [74,75]. In cancer cells, the inhibitors of $\mathrm{m}$ TORCI are of interest as inhibitors of cellular metabolism (temsirolimus and everolimus) [76].

\section{GENE THERAPY}

Current treatment strategies in mitochondrial disorders are largely supportive rather than curative. Gene therapy in the future may prove to be a deciding factor and completely change the approach to treat 
mitochondrial dysfunctional disorders. Such techniques may target the mitochondrial genome in oocyte [77-80].

The most prominent gene therapy is mitochondrial replacement therapy (MRT), aims to prevent the transmission of heritable disorders caused by mutations in the mitochondrial genome. MRT is a pre-conception intervention that involves replacing the pathogenic mitochondrial DNA of an embryo with healthy mitochondrial DNA from a donor.

Targeting the mitochondria in mitochondrial diseases with the restriction endonuclease SmaI enzyme has shown elimination of the mutant mtDNA followed with an increase in wild-type mtDNA and restoration of normal ATP levels [81].

\section{NON-PHARMACOLOGICAL MANAGEMENT}

\section{Physical exercise}

Mitochondria provide energy as ATP for muscle function. In mitochondrial dysfunctional states, there is less energy production coupled with increased excessive ROS, and this can trigger muscle atrophy, weakness, and loss of endurance. Exercise is known to increase mitochondria ETC activity in older human skeletal muscle, especially in sub sarcolemma (SS) mitochondria [82]. Exercise counters mitochondrial dysfunction. The compensatory nature of exercise is through pathways involving molecular signaling to transcription, as well as to post-transcriptional events within the mitochondrial synthesis and degradation [83]

\section{Healthy nutrition}

Malnutrition states such as anorexia, starvation, and cachexia result in SMD. Malnutrition leads to OXPHOS abnormalities. Improvement in intake of calories improves mitochondrial health in these patients $[84,85]$.

Natural antioxidants can prevent and treat the disorders related to defective mitochondria as age progresses. Ginkgo biloba, a herbal drug, used for the improvement of cognitive dysfunction. Curcumin a yellow pigment derived from the rhizome part of the turmeric plant; omega- 3 polyunsaturated fatty acids ( $\omega$-3 PUFAs), a group of essential fatty acids and triterpenoids, the derivatives of oleanolic acid are all known to inhibit oxidative stress [86].

\section{AVOIDING MITOCHONDRIAL TOXIC DRUGS}

There is number of drugs those have proven mitochondrial toxic effects and such drug should be avoided in mitochondrial dysfunctional disorders. These mitochondrial toxic drugs cause inhibition of ETC in OXPHOS, inducing oxidative stress, or inhibiting DNA replication, transcription, or translation [87].

Valproic acid (VPA) is a commonly used antiepileptic drug that has well-established hepatotoxicity and steatosis due to mitochondrial dysfunction. It selectively affects $\alpha$-lipoamide dehydrogenase in liver. Amide analogs of VPA show inhibitory effects on mitochondrial OXPHOS [88-90]. VPA produces inhibition of fatty acid oxidation, the citric acid cycle, OXPHOS, complex IV, and results in carnitine depletion [91].

Antiretroviral drugs cause impairment of mtDNA replication resulting in mtDNA depletion; carnitine deficiency, nucleoside reverse transcriptase inhibitors block both HIV reverse transcriptase and mitochondrial DNA polymerase gamma, the latter cause of the adverse effects due to these drugs. Mitochondrial toxicity includes defects in mitochondria DNA replication [92-96].

Statins are known for their efficacy in dyslipidemias but impair mitochondria. Statins cause abnormal mitochondrial morphology, decreased OXPHOS and activate apoptotic pathway, and deficiency of coenzyme Q10 [97]. Statin-induced mitochondrial dysfunction is the most likely cause of statin-associated muscle disease $[98,99]$.
Aminoglycosides antimicrobials are known for hearing loss, renal and cardic toxicity. They are mitochondrial toxic and impair mtDNA translation $[100,101]$.

Aspirin Reye's syndrome associated with aspirin is well-established. Aspirin leads to inhibition and uncoupling of OXPHOS. Aspirin causes an increase in ROS production, loss of mitochondrial membrane potential, and inhibition of mitochondrial respiratory functions [102]. Aspirin increases mitochondrial long-chain fatty acid oxidation and mitochondrial protein acetylation [103].

Acetaminophen is widely prescribed as an analgesic and antipyretic. However, its overdose can cause hepatotoxicity. Excess formation of the reactive metabolite $\mathrm{N}$-acetyl-p-benzoquinone imine leads to hepatic glutathione depletion. Oxidative stress and cessation of ATP synthesis occur. It causes nuclear DNA fragmentation and extensive mitochondrial dysfunction result in necrotic cell death [104,105]. Mitochondrial respiratory complex I is affected by a hepato-toxic effect of a high dose of acetaminophen [106]. High dose hepatotoxicity causes an increase in ROS levels and loss of glutathione. Increased oxidative stress impairs mitochondrial function involving permeability transition, resulting in loss of mitochondrial membrane potential and ability to synthesize ATP [107].

Metformin is an antidiabetic drug used in non-insulin-dependent diabetes mellitus. It inhibits mitophagy and increases the load of mutant mitochondrial DNA and also reduces oxidative stress [108].

\section{CONCLUSIONS}

Mitochondria are well known for their ability to synthesize ATP besides, the generation of ROS. In dysfunction of mitochondria, the energy depletion and accumulation of oxidants are likely outcome. The vital organs that exclusively depend on ATP for their function suffer most. Oxidative stress is involved in the pathophysiology of various disorders especially involving neurons and cardiovascular system. Mitochondrialtargeted pharmaceuticals and lifestyle modifications (healthy diet and regular exercise) are useful in these disorders.

\section{AUTHORS' CONTRIBUTIONS}

All authors contributed equally.

\section{CONFLICTS OF INTEREST}

The authors declared no conflicts of interest related to the study.

\section{AUTHORS' FUNDING}

The authors did not receive any funding for the research work.

\section{REFERENCES}

1. Chinnery PF. Mitochondrial disorders overview. In: Adam MP, Ardinger HH, Pagon RA, editors. GeneReviews ${ }^{\circledR}$. Seattle, WA: University of Washington, Seattle 1993-2021.

2. Niyazov DM, Kahler SG, Frye RE. Primary mitochondrial disease and secondary mitochondrial dysfunction: Importance of distinction for diagnosis and treatment. Mol Syndromol 2016;7:122-37.

3. Khan NA, Govindaraj P, Meena AK, Thangaraj K. Mitochondrial disorders: Challenges in diagnosis treatment. Indian $\mathrm{J}$ Med Res 2015;141:13-26.

4. Avula S, Parikh S, Demarest S, Kurz J, Gropman A. Treatment of mitochondrial disorders. Curr Treat Options Neurol 2014;16:292.

5. Quinzii CM, Hirano M. Coenzyme Q and mitochondrial disease. Dev Disabil Res Rev 2010;16:183-8.

6. Hargreaves IP. Coenzyme Q10 as a therapy for mitochondrial disease. Int J Biochem Cell Biol 2014;49:105-11.

7. Orsucci D, Mancuso M, Ienco EC, LoGerfo A, Siciliano G. Targeting mitochondrial dysfunction and neurodegeneration by means of coenzyme Q10 and its analogues. Curr Med Chem 2011;18:4053-64.

8. Littarru GP, Tiano L. Clinical aspects of coenzyme Q10: An update. Nutrition 2010;26:250-4 
9. Graham D, Huynh NN, Hamilton CA, Beattie E, Smith RA, Cochemé HM, et al. mitochondria-targeted antioxidant MitoQ10 improves endothelial function and attenuates cardiac hypertrophy. Hypertension 2009; 54:322-8.

10. Isobe C, Abe T, Terayama Y. Levels of reduced and oxidized coenzyme Q-10 and 8-hydroxy-2'-deoxyguanosine in the cerebrospinal fluid of patients with living Parkinson's disease demonstrate that mitochondrial oxidative damage and/or oxidative DNA damage contributes to the neurodegenerative process. Neurosci Lett 2010;469:159-63.

11. Littarru GP, Langsjoen P. Coenzyme Q10 and statins: Biochemical and clinical implications. Mitochondrion 2007;7 Suppl:S168-74.

12. Tauskela JS. MitoQ--a mitochondria-targeted antioxidant. IDrugs 2007:10:399-412.

13. Smith RA, Porteous CM, Coulter CV, Murphy MP. Selective targeting of an antioxidant to mitochondria. Eur J Biochem 1999;263:709-16.

14. Adlam VJ, Harrison JC, Porteous CM, James AM, Smith RA, Murphy MP, et al. Targeting an antioxidant to mitochondria decreases cardiac ischemia-reperfusion injury. FASEB J 2005;19:1088-95.

15. McLachlan J, Beattie E, Murphy MP, Koh-Tan CH, Olson E, Beattie W, et al. Combined therapeutic benefit of mitochondriatargeted antioxidant, MitoQ10, and angiotensin receptor blocker, losartan, on cardiovascular function. J Hypertens 2014;32:555-64.

16. Ng LF, Gruber J, Cheah IK, Goo CK, Cheong WF, Shui G, et al. The mitochondria-targeted antioxidant MitoQ extends lifespan and improves healthspan of a transgenic Caenorhabditis elegans model of Alzheimer disease. Free Radic Biol Med 2014;71:390-401.

17. Trnka J, Blaikie FH, Smith RA, Murphy MP. A mitochondriatargeted nitroxide is reduced to its hydroxylamine by ubiquinol in mitochondria. Free Radic Biol Med 2008;44:1406-19.

18. Dey S, DeMazumder D, Sidor A, Foster DB, O'Rourke B. Mitochondrial ROS drive sudden cardiac death and chronic proteome remodeling in heart failure. Circ Res 2018;123:356-71

19. Choumar A, Tarhuni A, Lettéron P, Reyl-Desmars F, Dauhoo N, Damasse J, et al. Lipopolysaccharide-induced mitochondrial DNA depletion. Antioxid Redox Signal 2011;15:2837-54

20. Dikalova AE, Bikineyeva AT, Budzyn K, Nazarewicz RR, McCann $\mathrm{L}$, Lewis $\mathrm{W}$, et al. Therapeutic targeting of mitochondrial superoxide in hypertension. Circ Res 2010;107:106-16.

21. Patil NK, Parajuli N, MacMillan-Crow LA, Mayeux PR. Inactivation of renal mitochondrial respiratory complexes and manganese superoxide dismutase during sepsis: Mitochondriatargeted antioxidant mitigates injury. Am J Physiol Renal Physiol 2014;306:F734-43

22. Wang A, Keita $̊ \mathrm{~V}$, Phan V, McKay CM, Schoultz I, Lee J, et al. Targeting mitochondria-derived reactive oxygen species to reduce epithelial barrier dysfunction and colitis. Am J Pathol 2014;184:251627.

23. Pandey M, Verma RK, Saraf SA. Neurtaceuticals new era of medicine and health. Asian J Pharm Clin Res 2010;5:11-5.

24. Mosegaard S, Dipace G, Bross P, Carlsen J, Gregersen N, Olsen RKJ. Riboflavin deficiency-implications for general human health and inborn errors of metabolism. Int J Mol Sci 2020;21:3847.

25. Balasubramaniam S, Lee YJ. Riboflavin metabolism: Role in mitochondrial functions. J Transl Genet Genom 2020;4:285-306.

26. Udhayabanu T, Manole A, Rajeshwari M, Varalakshmi P, Houlden H, Ashokkumar B. Riboflavin responsive mitochondrial dysfunction in neurodegenerative diseases. J Clin Med 2017:6:52

27. Bugiani M, Lamantea E, Invernizzi F, Moroni I, Bizzi A, Zeviani M, et al. Effects of riboflavin in children with complex II deficiency. Brain Dev 2006;28:576-81.

28. Al Jasmi F, Al Zaabi N, Al-Thihli K, Al Teneiji AM, Hertecant J, El-Hattab AW. Endothelial dysfunction and the effect of arginine and citrulline supplementation in children and adolescents with mitochondrial diseases. J Cent Nerv Syst Dis 2020;12:1179573520909377.

29. El-Hattab AW, Emrick LT, Chanprasert S, Craigen WJ, Scaglia F. Mitochondria: Role of citrulline and arginine supplementation in MELAS syndrome. Int J Biochem Cell Biol 2014;48:85-91.

30. Quijada-Fraile P, O'Callaghan M, Martín-Hernández E, Montero R, Garcia-Cazorla À, de Aragón AM, et al. Follow-up of folinic acid supplementation for patients with cerebral folate deficiency and Kearns-Sayre syndrome. Orphanet J Rare Dis 2014;9:217.

31. Ramaekers VT, Weis J, Sequeira JM, Quadros EV, Blau N. Mitochondrial complex I encephalomyopathy and cerebral 5-methyltetrahydrofolate deficiency. Neuropediatrics 2007;38:184-7.
32. Ormazabal A, Casado M, Molero-Luis M, Montoya J, Rahman S, Aylett SB, et al. Can folic acid have a role in mitochondrial disorders? Drug Discov Today 2015;20:1349-54.

33. Birkmayer JG, Vrecko C, Volc D, Birkmayer W. Nicotinamide adenine dinucleotide (NADH)--a new therapeutic approach to Parkinson's disease. Comparison of oral and parenteral application. Acta Neurol Scand Suppl 1993;146:32-5.

34. Birkmayer JG. Coenzyme nicotinamide adenine dinucleotide: New therapeutic approach for improving dementia of the Alzheimer type. Ann Clin Lab Sci 1996;26:1-9.

35. Forsyth LM, Preuss HG, MacDowell AL, Chiazze L Jr, Birkmayer GD, Bellanti JA. Therapeutic effects of oral NADH on the symptoms of patients with chronic fatigue syndrome. Ann Allergy Asthma Immunol 1999;82:185-91.

36. Wang G, Han T, Nijhawan D, Theodoropoulos P, Naidoo J, Yadavalli $\mathrm{S}$, et al. $\mathrm{P} 7 \mathrm{C} 3$ neuroprotective chemicals function by activating the rate-limiting enzyme in NAD salvage. Cell 2014;158:1324-34.

37. Thirunavukkarasu M, Penumathsa SV, Koneru S, Juhasz B, Zhan L, Otani $\mathrm{H}$, et al. Resveratrol alleviates cardiac dysfunction in streptozotocin-induced diabetes: Role of nitric oxide, thioredoxin, and heme oxygenase. Free Radic Biol Med 2007;43:720-9.

38. Khan NA, Auranen M, Paetau I, Pirinen E, Euro L, Forsström S, et al. Effective treatment of mitochondrial myopathy by nicotinamide riboside, a Vitamin B3. EMBO Mol Med 2014;6:721-31.

39. Nicolson GL. Metabolic syndrome and mitochondrial function: Molecular replacement and antioxidant supplements to prevent membrane peroxidation and restore mitochondrial function. J Cell Biochem 2007;100:1352-69.

40. Nicolson GL, Ellithorpe R. Lipid replacement and antioxidant nutritional therapy for restoring mitochondrial function and reducing fatigue in chronic fatigue syndrome and other fatiguing illnesses. J Chronic Fatigue Syndr 2006;13:57-68

41. Reuter SE, Evans AM. Carnitine and acylcarnitines: Pharmacokinetic, pharmacological and clinical aspects. Clin Pharmacokinet 2012;51:553-72.

42. Rizos I. Three-year survival of patients with heart failure caused by dilated cardiomyopathy and L-carnitine administration. Am Heart J 2000;139:S120-3.

43. Serati AR, Motamedi MR, Emami S, Varedi P, Movahed MR. L-carnitine treatment in patients with mild diastolic heart failure is associated with improvement in diastolic function and symptoms. Cardiology 2010;116:178-82

44. Siasos G, Tousoulis D, Tsigkou V, Kokkou E, Oikonomou E, Vavuranakis M, et al. Flavonoids in atherosclerosis: An overview of their mechanisms of action. Curr Med Chem 2013;20:2641-60.

45. Testai L. Flavonoids and mitochondrial pharmacology: A new paradigm for cardioprotection. Life Sci 2015;135:68-76.

46. Loke WM, Proudfoot JM, Hodgson JM, McKinley AJ, Hime N, Magat M, et al. Specific dietary polyphenols attenuate atherosclerosis in apolipoprotein E-knockout mice by alleviating inflammation and endothelial dysfunction. Arterioscler Thromb Vasc Biol 2010;30:74957.

47. Priyanga SK, vijaylakshmi K. Investigation of antioxidant potential of quercetin and hesperidin: As in vitro approach. Asian J Pharm Clin Res 2017;10:83-86.

48. Scoditti E, Calabriso N, Massaro M, Pellegrino M, Storelli $\mathrm{C}$, Martines $\mathrm{G}$, et al. Mediterranean diet polyphenols reduce inflammatory angiogenesis through MMP-9 and COX-2 inhibition in human vascular endothelial cells: A potentially protective mechanism in atherosclerotic vascular disease and cancer. Arch Biochem Biophys 2012;527:81-9.

49. Natsume M, Baba S. Suppressive effects of cacao polyphenols on the development of atherosclerosis in apolipoprotein E-deficient mice. Subcell Biochem 2014;77:189-98.

50. Michels AJ, Frei B. Myths, artifacts, and fatal flaws: Identifying limitations and opportunities in Vitamin $\mathrm{C}$ research. Nutrients 2013;5:5161-92

51. Cheng J, Kamiya K, Kodama I. Carvedilol: Molecular and cellular basis for its multifaceted therapeutic potential. Cardiovasc Drug Rev 2001;19:152-71

52. de Cavanagh EM, Toblli JE, Ferder L, Piotrkowski B, Stella I, Inserra F. Renal mitochondrial dysfunction in spontaneously hypertensive rats is attenuated by losartan but not by amlodipine. Am J Physiol Regul Integr Comp Physiol 2006;290:R1616-25.

53. Parihar A, Parihar MS, Zenebe WJ, Ghafourifar P. Statins lower calcium-induced oxidative stress in isolated mitochondria. Hum Exp 
Toxicol 2012;31:355-63

54. Hernanz R, Martín Á, Pérez-Girón JV, Palacios R, Briones AM, Miguel M, et al. Pioglitazone treatment increases COX--derived prostacyclin production and reduces oxidative stress in hypertensive rats: Role in vascular function. Br J Pharmacol 2012;166:1303-19.

55. Sharov VG, Todor A, Khanal S, Imai M, Sabbah HN. Cyclosporine A attenuates mitochondrial permeability transition and improves mitochondrial respiratory function in cardiomyocytes isolated from dogs with heart failure. J Mol Cell Cardiol 2007;42:150-8.

56. Zhang WH, Wang H, Wang X, Narayanan MV, Stavrovskaya IG, Kristal BS, et al. Nortriptyline protects mitochondria and reduces cerebral ischemia/hypoxia injury. Stroke 2008;39:455-62.

57. Lombard DB, Tishkoff DX, Bao J. Mitochondrial sirtuins in the regulation of mitochondrial activity and metabolic adaptation. Handb Exp Pharmacol 2011;206:163-88.

58. Osborne B, Bentley NL, Montgomery MK, Turner N. The role of mitochondrial sirtuins in health and disease. Free Radic Biol Med 2016;100:164-74.

59. Lagouge M, Argmann C, Gerhart-Hines Z, Meziane H, Lerin C, Daussin $\mathrm{F}$, et al. Resveratrol improves mitochondrial function and protects against metabolic disease by activating SIRT1 and PGC1alpha. Cell 2006;127:1109-22.

60. Saiko P, Szakmary A, Jaeger W, Szekeres T. Resveratrol and its analogs: Defense against cancer, coronary disease and neurodegenerative maladies or just a fad? Mutat Res 2008;658:68-94.

61. Parikh S, Saneto R, Falk MJ, Anselm I, Cohen BH, Haas R, et al. A modern approach to the treatment of mitochondrial disease. Curr Treat Options Neurol 2009;11:414-30.

62. Ungvari Z, Sonntag WE, de Cabo R, Baur JA, Csiszar A. Mitochondrial protection by resveratrol. Exerc Sport Sci Rev 2011;39:128-32.

63. Csiszar A, Labinskyy N, Pinto JT, Ballabh P, Zhang H, Losonczy $\mathrm{G}$, et al. Resveratrol induces mitochondrial biogenesis in endothelial cells. Am J Physiol Heart Circ Physiol 2009;297:H13-20.

64. Wang H, Guan Y, Widlund AL, Becker LB, Baur JA, Reilly PM, et al. Resveratrol ameliorates mitochondrial dysfunction but increases the risk of hypoglycemia following hemorrhagic shock. J Trauma Acute Care Surg 2014;77:926-33.

65. Bellaver B, Bobermin LD, Souza DG, Rodrigues MD, de Assis AM, Wajner M, et al. Signaling mechanisms underlying the glioprotective effects of resveratrol against mitochondrial dysfunction. Biochim Biophys Acta 2016;1862:1827-38.

66. Augustyniak J, Lenart J, Gaj P, Kolanowska M, Jazdzewski K, Stepien PP, et al. Bezafibrate upregulates mitochondrial biogenesis and influence neural differentiation of human-induced pluripotent stem cells. Mol Neurobiol 2019;56:4346-63.

67. Steele H, Gomez-Duran A, Pyle A, Hopton S, Newman J, Stefanetti $\mathrm{RJ}$, et al. Metabolic effects of bezafibrate in mitochondrial disease. EMBO Mol Med 2020;12:e11589.

68. Kumamoto University. Determining the Cause of Difficult-tocontrol Mitochondrial Diseases. ScienceDaily; 2018. http://www. sciencedaily.com/rele ases/2018/03/180307112740.htm

69. Fakruddin M, Wei FY, Suzuki T, Asano K, Kaieda T, Omori A, et al. Defective mitochondrial tRNA taurine modification activates global proteostress and leads to mitochondrial disease. Cell Rep 2018;22:482-96

70. Schaffer S, Kim HW. Effects and mechanisms of taurine as a therapeutic agent. Biomol Ther (Seoul) 2018;26:225-41

71. Ohsawa Y, Hagiwara H, Nishimatsu SI, Hirakawa A, Kamimura N, Ohtsubo $\mathrm{H}$, et al. Taurine supplementation for prevention of strokelike episodes in MELAS: A multicentre, open-label, 52-week phase III trial. J Neurol Neurosurg Psychiatry 2019;90:529-36.

72. Xu S, He M, Zhong M, Li L, Lu Y, Zhang Y, et al. The neuroprotective effects of taurine against nickel by reducing oxidative stress and maintaining mitochondrial function in cortical neurons. Neurosci Lett 2015;590:52-7.

73. Reddy PH. Inhibitors of mitochondrial fission as a therapeutic strategy for diseases with oxidative stress and mitochondrial dysfunction. $\mathrm{J}$ Alzheimers Dis 2014;40:245-56.

74. Morita M, Gravel SP, Chénard V, Sikström K, Zheng L, Alain T, et al. mTORC1 controls mitochondrial activity and biogenesis through 4E-BP-dependent translational regulation. Cell Metab 2013;18:698711.

75. Morita M, Prudent J, Basu K, Goyon V, Katsumura S, Hulea L, et al. mTOR controls mitochondrial dynamics and cell survival via MTFP1. Mol Cell 2017;67:922-3500000.

76. de la Cruz López KG, Guzmán ME, Sánchez EO, Carrancá AG.
mTORC1 as a regulator of mitochondrial functions and a therapeutic target in cancer. Front Oncol 2019;9:1373.

77. Tachibana M, Sparman M, Sritanaudomchai H, Ma H, Clepper $\mathrm{L}$, Woodward $\mathrm{J}$, et al. Mitochondrial gene replacement in primate offspring and embryonic stem cells. Nature 2009;461:367-72.

78. DiMauro S, Mancuso M. Mitochondrial diseases: Therapeutic approaches. Biosci Rep 2007;27:125-37.

79. Doyle SR, Chan CK. Mitochondrial gene therapy: An evaluation of strategies for the treatment of mitochondrial DNA disorders. Hum Gene Ther 2008;19:1335-48.

80. Agresti CA, Halkiadakis PN, Tolias P. MERRF and MELAS: Current gene therapy trends and approaches. J Transl Genet Genom 2018;2:9.

81. Tanaka M, Borgeld HJ, Zhang J, Muramatsu S, Gong JS, Yoneda M, et al. Gene therapy for mitochondrial disease by delivering restriction endonuclease SmaI into mitochondria. J Biomed Sci 2002;9:534-41.

82. Menshikova EV, Ritov VB, Fairfull L, Ferrell RE, Kelley DE, Goodpaster $\mathrm{BH}$. Effects of exercise on mitochondrial content and function in aging human skeletal muscle. J Gerontol A Biol Sci Med Sci 2006;61:534-40.

83. Oliveira AN, Hood DA. Exercise is mitochondrial medicine for muscle. Sports Med Health Sci 2019;1:11-8.

84. Wortmann SB, Zweers-van Essen H, Rodenburg RJ, van den Heuvel LP, de Vries MC, Rasmussen-Conrad E, et al. Mitochondrial energy production correlates with the age-related BMI. Pediatr Res 2009;65:103-8.

85. Morava E, Rodenburg R, van Essen HZ, De Vries M, Smeitink J. Dietary intervention and oxidative phosphorylation capacity. J Inherit Metab Dis 2006;29:589.

86. Kumar A, Singh A. A review on mitochondrial restorative mechanism of antioxidants in Alzheimer's disease and other neurological conditions. Front Pharmacol 2015;6:206.

87. Chan K, Truong D, Shangari N, O'Brien PJ. Drug-induced mitochondrial toxicity. Expert Opin Drug Metab Toxicol 2005;1:65569.

88. Kudin AP, Mawasi H, Eisenkraft A, Elger CE, Bialer M, Kunz WS. Mitochondrial liver toxicity of valproic acid and its acid derivatives is related to inhibition of $\alpha$-lipoamide dehydrogenase. Int J Mol Sci 2017;18:1912.

89. Salsaa M, Pereira B, Liu J, Yu W, Jadhav S, Hüttemann M, et al. Valproate inhibits mitochondrial bioenergetics and increases glycolysis in Saccharomyces cerevisiae. Sci Rep 2020;10:11785.

90. Caiment F, Wolters J, Smit E, Schrooders Y, Kleinjans J, van den Beucken T. Valproic acid promotes mitochondrial dysfunction in primary human hepatocytes in vitro; impact of $\mathrm{C} / \mathrm{EBP} \alpha$-controlled gene expression. Arch Toxicol 2020;94:3463-73.

91. Silva MF, Aires CC, Luis PB, Ruiter JP, IJlst L, Duran M, et al. Valproic acid metabolism and its effects on mitochondrial fatty acid oxidation: A review. J Inherit Metab Dis 2008;31:205-16.

92. Kakuda TN. Pharmacology of nucleoside and nucleotide reverse transcriptase inhibitor-induced mitochondrial toxicity. Clin Ther 2000;22:685-708.

93. Kohler JJ, Lewis W. A brief overview of mechanisms of mitochondrial toxicity from NRTIs. Environ Mol Mutagen 2007:48:166-72.

94. Dalakas MC. Peripheral neuropathy and antiretroviral drugs. J Peripher Nerv Syst 2001;6:14-20.

95. Scruggs ER, Dirks Naylor AJ. Mechanisms of zidovudine-induced mitochondrial toxicity and myopathy. Pharmacology 2008;82:83-8.

96. Pinti M, Salomoni P, Cossarizza A. Anti-HIV drugs and the mitochondria. Biochim Biophys Acta 2006;1757:700-7.

97. Broniarek I, Jarmuszkiewicz W. Statins and mitochondria. Postepy Biochem 2016;62:77-84.

98. Ramachandran R, Wierzbicki AS. Statins, muscle disease and mitochondria. J Clin Med 2017;6:75.

99. Wagner BK, Kitami T, Gilbert TJ, Peck D, Ramanathan A, Schreiber SL, et al. Large-scale chemical dissection of mitochondrial function. Nat Biotechnol 2008;26:343-51.

100. Bindu LH, Reddy PP. Genetics of aminoglycoside-induced and prelingual non-syndromic mitochondrial hearing impairment: A review. Int J Audiol 2008;47:702-7.

101. Fischel-Ghodsian N. Genetic factors in aminoglycoside toxicity. Ann N Y Acad Sci 1999;884:99-109.

102. Raza H, John A. Implications of altered glutathione metabolism in aspirin-induced oxidative stress and mitochondrial dysfunction in HepG2 cells. PLoS One 2012;7:e36325.

103. Uppala R, Dudiak B, Beck ME, Bharathi SS, Zhang Y, Stolz DB, et al. Aspirin increases mitochondrial fatty acid oxidation. Biochem 
Biophys Res Commun 2017;482:346-51.

104. Burke AS, MacMillan-Crow LA, Hinson JA. Reactive nitrogen species in acetaminophen-induced mitochondrial damage and toxicity in mouse hepatocytes. Chem Res Toxicol 2010;23:1286-92.

105. Jaeschke H, Duan L, Nguyen N, Ramachandran A. Mitochondrial damage and biogenesis in acetaminophen-induced liver injury. Liver Res 2019;3:150-6.

106. Chrøis KM, Larsen S, Pedersen JS, Rygg MO, Boilsen AE,
Bendtsen F, et al. Acetaminophen toxicity induces mitochondrial complex I inhibition in human liver tissue. Basic Clin Pharmacol Toxicol 2019; 126:86-91.

107. Hinson JA, Roberts DW, James LP. Mechanisms of acetaminopheninduced liver necrosis. Handb Exp Pharmacol 2010;196:369-405.

108. Spiller HA, Sawyer TS. Toxicology of oral antidiabetic medications. Am J Health Syst Pharm 2006;63:929-38. 